\title{
Microfluidics/CMOS orthogonal capabilities for cell biology
}

\section{Journal Article}

\section{Author(s):}

Linder, Vincent; Koster, Sander; Franks, Wendy; Kraus, Tobias; Verpoorte, Elisabeth; Heer, Flavio; Hierlemann, Andreas (i); De Rooij, Nicolaas F.

\section{Publication date:}

2006-06

\section{Permanent link:}

https://doi.org/10.3929/ethz-b-000412932

\section{Rights / license:}

In Copyright - Non-Commercial Use Permitted

\section{Originally published in:}

Biomedical Microdevices 8(2), https://doi.org/10.1007/s10544-006-7711-9 


\title{
Microfluidics/CMOS orthogonal capabilities for cell biology
}

\author{
Vincent Linder · Sander Koster · Wendy Franks · Tobias Kraus · Elisabeth Verpoorte • Flavio Heer • \\ Andreas Hierlemann • Nico F. de Rooij
}

(C) Springer Science + Business Media, LLC 2006

\begin{abstract}
The study of individual cells and cellular networks can greatly benefit from the capabilities of microfabricated devices for the stimulation and the recording of electrical cellular events. In this contribution, we describe the development of a device, which combines capabilities for both electrical and pharmacological cell stimulation, and the subsequent recording of electrical cellular activity. The device combines the unique advantages of integrated circuitry (CMOS technology) for signal processing and microfluidics for drug delivery. Both techniques are ideally suited to study electrogenic mammalian cells, because feature sizes are of the same order as the cell diameter, $\sim 50 \mu \mathrm{m}$. Despite these attractive features, we observe a size mismatch between microfluidic devices, with bulky fluidic connections to the outside world, and highly miniaturized CMOS chips. To overcome this problem, we developed a microfluidic flow cell that accommodates a small CMOS chip. We simulated the performances of a flow cell based on a 3-D microfluidic system, and then fabricated the device to experimentally verify the nutrient delivery and localized drug delivery performance. The flow-cell has a constant nutrient flow, and six drug inlets that can individually deliver a drug to the cells. The experimental analysis of the nutrient and drug flow mass transfer properties in the flowcell are in good agreement with our
\end{abstract}

V. Linder · T. Kraus · N. F. de Rooij $(\square)$

SAMLAB, Institute of Microtechnology, University of Neuchâtel, Switzerland

e-mail: nico.derooij@unine.ch

S. Koster · E. Verpoorte

SAMLAB, Institute of Microtechnology, University of Neuchâtel, Switzerland; Present address: Groningen Research Institute of Pharmacy, University of Groningen, the Netherlands

W. Franks · F. Heer · A. Hierlemann

ETH Zurich, Physical Electronics Laboratory, Zürich, Switzerland simulations. For an experimental proof-of-principle, we successfully delivered, in a spatially resolved manner, a 'drug' to a culture of HL-1 cardiac myocytes.

\section{Introduction}

Merging the complementary capabilities of electronic chips and microfluidics will greatly benefit the field of cell biology. On one hand, electronic chips provide high density arrays of transducers for electrical recording and stimulation, and, on the other hand, microfluidics offer automated fluid handling capabilities and tools for the delivery of small, precise quantities of chemicals. Both techniques have feature sizes in the micrometer scale, and are ideally suited to study cell-based systems, such as mammalian cells which typically have a diameter of 10-50 $\mu \mathrm{m}$. By combining these techniques, the response of cellular electrically activity to chemical and electrical stimulation cues can be thoroughly investigated.

Microelectrode arrays (MEA) are very useful research tools for studying electroactive cells, such as neurons and cardiomyocytes. Since its inception in the early 1970s (Thomas et al., 1972) most devices were based on electrically conductive lines patterned on glass (typically palladium, gold (Gross et al., 1995) or indium tin oxide (Gross et al., 1985)), and are increasingly becoming a standard tool for research in cell biology (Keefer et al., 2001; Tscherter et al., 2001; Streit et al., 2001; Jimbo and Robinson, 2000). One current limitation of these microelectrode arrays is the difficulty to connect all the electrodes to the off-chip electronics since each electrode requires a dedicated interconnect to the outside world. The array size is therefore limited by the available space on the perimeter of the chip that can be used for interconnects. Moreover, using off-chip electronics 


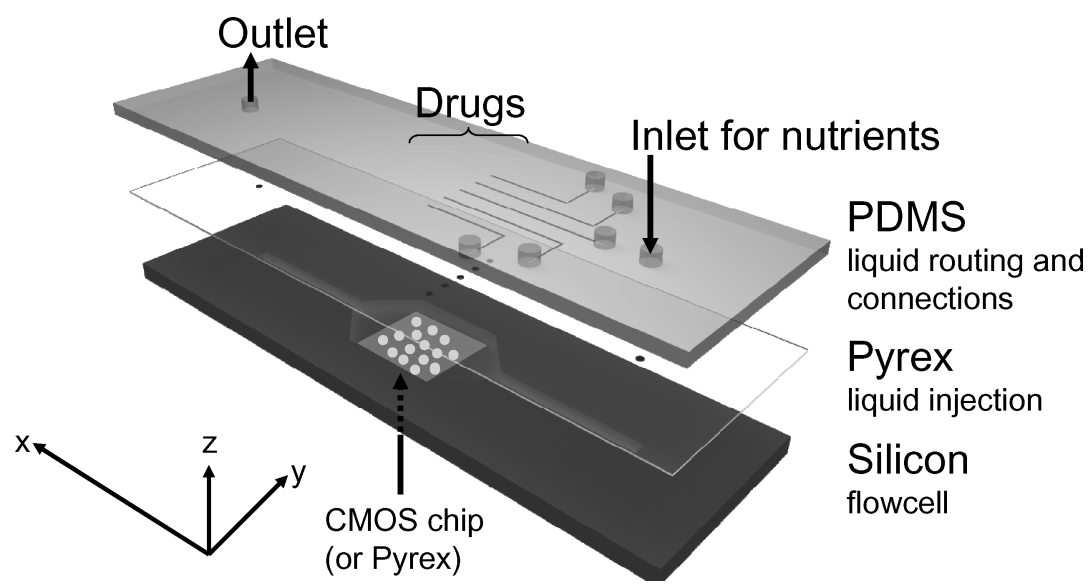

Fig. 1 Three-dimensional representation of the flow cell. A micromachined silicon substrate is etched to form the flowcell. In the central part, a hole is etched through the wafer to later accommodate the CMOS chip. At each side of the cavity, channels are formed for the flow of nutrients across the cavity. The cells are grown in the chamber (i.e., on the surface

for signal processing, the electrical signal will be deleteriously affected by noise contributions occurring during the transfer of the signal from the MEA to the external filtering and amplifying electronics. These limitations highlight to the need for an electronic chip with both (i) high density arrays of sensing electrodes, and (ii) on-chip electronics for signal processing. The use of CMOS (complementary metal oxide semiconductor) technology fulfills these technical requirements (Bai and Wise, 2001; DeBusschere and Kovacs, 2001; Eversmann et al., 2004).

Microfluidic systems have become very popular for many bioassays. Two attractive features of microfluidics are especially useful for cellular assays: (i) the possibility to automate nutrient delivery, (Tourovskaia et al., 2005; Hung et al., 2004) and (ii) the capability of directing minute quantities of liquid to the cells with high precision (Kaji et al., 2003; Takayama et al., 2003; Matsubara et al., 2004). These microfluidic devices need millimeter-scale fluidic connections and/or reservoirs of reagents to ensure the operation of the chip. As a result, microfluidic chips typically have micrometer-sized channels and chambers, but generally cover areas of several square centimeters to allow sufficient space for the connections to the outside world.

The combination of CMOS technology and microfluidics will result in a powerful tool, however their relative sizes are mismatched: CMOS chips are cost efficient only if their area is kept to minimum (several square millimeters), while microfluidic systems require areas much larger than that of CMOS. For microfluidic devices that carry out complex tasks (i.e., localized drug delivery), the area requirements increase further because of the large number of necessary fluidic connections to perform these complex tasks. Quake et al. have, for example, integrated several thousand $\mathrm{nL}$-scale-reactors on a single microfluidic chip that had a size between 20 and of the CMOS chip). A drilled cover plate in Pyrex closes the flowcell. Nutrients and drug are delivered inside the device through holes and channels in the PDMS substrate. The coordinate system depicted in the bottom left part of the scheme defines the directions of the axes used in the following figures

$30 \mathrm{~cm}^{2}$ where each reactor measured several $\mathrm{mm}^{2}$. This is still many orders of magnitude larger in size than CMOS chips where $\mu \mathrm{m}$-sized functionalities can be integrated (Unger et al., 2000; Liu et al., 2003).

In this contribution, we present a microfluidic device that can accommodate a diced CMOS MEA. The flow cell covers an area large enough for simple fluidic connections to the outside world, with a cavity at the core of the device for accomodating a CMOS chip. Using a 3-D channel layout, we performed simple tasks such as nutrient dispensing, and more complex tasks such as drug assays. We modeled the

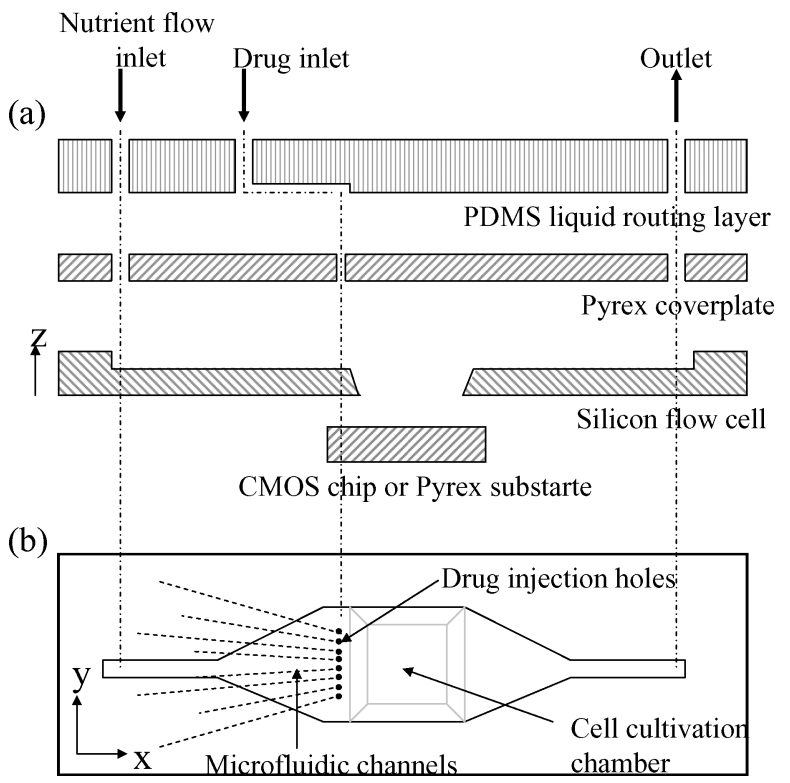

Fig. 2 Schematic representation of the flowcell used for flow simulation. The bottom part of the cavity is closed with a Pyrex wafer (by anodic bonding), all other parts are identical to that described in the caption of Fig. 1. (a) Side view and (b) top view 
flows in the microfluidic flow cell with simulation software, and then experimentally demonstrated the capability of the device for applications in cell biology by locally staining the HL-1 cardiac myocyte cell line with DNA dyes.

\section{Results and discussion}

For the fabrication of the microfluidic device, we selected a methodology based on silicon micromachining. The rationale of this approach is to physically separate the fluid handling from the electronics. Starting with a silicon wafer, we used one side of the wafer for implementing the microfluidic components (i.e., channels, fluidic connections to the outside world), and the other side of the wafer to connect to the CMOS chip. The silicon wafer is etched through to enable communication between both worlds (see the schematic representation in Fig. 1 and Fig. 2). Applications in cell biology often require visual observations of the cells with regular microscopes. For our device, we used transparent materials to seal the flow cell above the cavity, so that the surface of the CMOS chip remains visible at any time. This approach enables the user to apply the same protocols of optical observation as are used in conventional bioassays.

We designed the overall shape of the flow cell for manufacturability with standard micromachining processes. To avoid
Fig. 3 Localized drug delivery to cells. (a) Schematic side- and top-view of the drug delivery. A nutrient flow of nutrient $\left(20-100 \mu \mathrm{L} \cdot \mathrm{min}^{-1}\right)$ is applied from the inlet to the outlet, and the drug is injected downwards into the flow of nutrient at the entrance of the cavity. The drug shows a laminar flow profile that reaches the cells on the bottom of the flow cell. (b)

Experimental characterization of the broadening of the drug streak, top view; a dye was used to illustrate drug delivery (a)
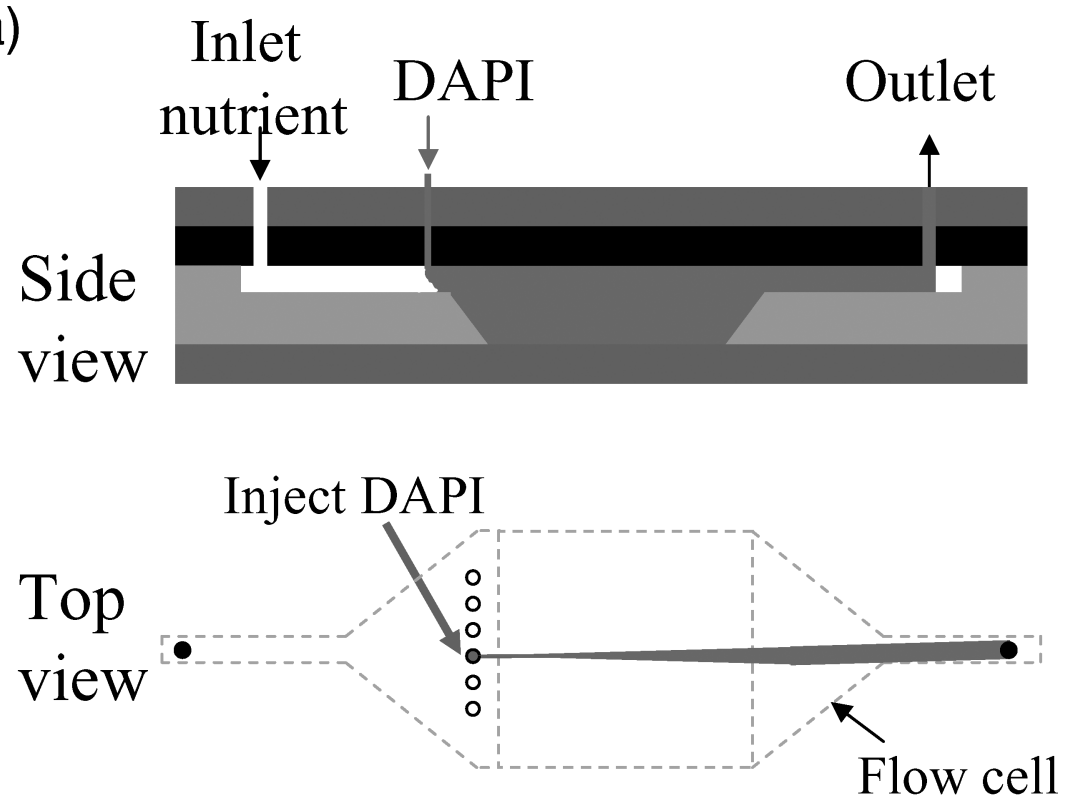

(b)

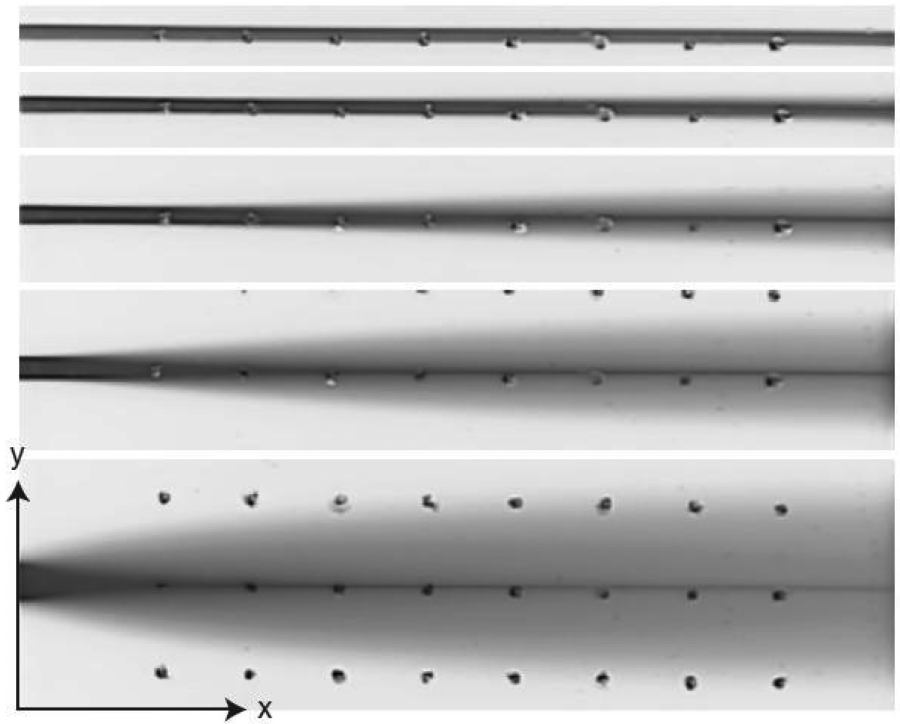

$100 \mu \mathrm{l} / \mathrm{min}$

$80 \mu \mathrm{l} / \mathrm{min}$

$60 \mu \mathrm{l} / \mathrm{min}$

$40 \mu \mathrm{l} / \mathrm{min}$

$20 \mu \mathrm{l} / \mathrm{min}$ 
the presence of square angles in the flow cell, we formed the channels and the cavity with $\mathrm{KOH}$ wet etching to obtain the characteristic angle of $54.7^{\circ}$ for the side walls (as illustrated in Fig. 2). The flow cell is composed of two channels, each $100 \mu \mathrm{m}$ deep, that widen until they reach the width of the cavity, and the cavity itself ( $525 \mu \mathrm{m}$ deep trough the wafer, and $6 \times 6 \mathrm{~mm}^{2}$ or $10 \times 10 \mathrm{~mm}^{2}$, depending on the design used). For the characterization of the flow cell, we sealed the lower part of the cavity with a Pyrex substrate, instead of bonding to the CMOS chip. The resulting geometry of the device is very similar regardless of the approach chosen (Pyrex or CMOS bottom). The upper part of the flow cell sealed with a transparent lid made of Pyrex glass and a layer of poly (dimethylsiloxane) (PDMS) containing molded microfluidic routing channels. Holes are drilled in the Pyrex substrate for liquid transport, with inlet and outlet holes aligned to the respective end of the channels that were etched in the silicon flowcell. An additional array of holes is available for the localized introduction of drug. The holes are located within the path of the nutrient, shortly before the entrance to the cavity (see also Fig. 2). The PDMS routing layer contains microchannels connecting the holes in the Pyrex wafer with corresponding reservoirs, which are adequately spatially separated to allow connection to the outside world. The elastomeric PDMS has the additional advantage of simplifying the connection of tubing with the microfluidic chip: we obtained leak-free connections by squeezing hard tubing into the reservoir, which has a slightly smaller diameter than the tubing.

Based on the geometry illustrated in Fig. 2, we carried out flow simulations using a model developed earlier (Kraus et al., 2005), which accounted for the gradually increasing cross-section of the inlet channel and the abrupt change in geometry of the cavity (i.e., the $54.7^{\circ}$ opening angle of the cell cultivation area). We showed that backward flows, that would disrupt the laminar flow of nutrient delivery, only occur at flow velocities far larger than those required. With flow rates of 10,100 and $1000 \mu \mathrm{L} \cdot \mathrm{min}^{-1}$ we calculated corresponding values of $0.05,0.3$ and 1 for the Reynolds numbers (Re). Backflows were predicted to appear only for Re larger than 50. Under high flow conditions, the shear forces acting on cells attached to the surface of the cavity could cause the cells to detach. We calculated that the stress produced by a flow rate of $300 \mu \mathrm{L} \cdot \mathrm{min}^{-1}$ resulted in a stress of $11 \mathrm{mPa}$ acting on the cells bound to the cavity surface (Kraus et al., 2005). This value is four orders of magnitude lower than that reported to lead to cell detachment due to shear flow (Lu et al., 2004).

We designed the flow cell in a way that we could exploit laminar flows to deliver minute amounts of chemical to a subset of cells in the cavity, see Fig. 3(a). Using the array of holes located next to the entrance of the cavity (see Fig. 2), we injected a drug solution from the top into the main flow of nutrient. The surface area of the cavity that was exposed to the drug could be modified by adjusting the ratio of nutrient to drug flow rates [Fig. 3(b)]: high nutrient flow rates led to narrow drug streaks of drug. In several experiments the parameters were determined to achieve defined widths of drug streaks. However, the top-view observations of the dye did not confirm that the drug injected from the top part of the flow cell travels all the way to the bottom to reach the immobilized cells.

We therefore experimentally demonstrated that the drug reaches the bottom of the flow cell, and hence the seeded cells, by performing a DNA staining experiment using the HL-1 cardiac myocytes cell line. We seeded and grew the cardiac myocytes in the cavity, and before closing the cavity with the Pyrex/PDMS cover, we fixed and permeated the cells to allow the DNA stain DAPI $\left(4^{\prime}, 6^{\prime}\right.$-diamidine-2 phylindole dihydrochloride) to permeate the membranes and reach the nuclei of the cells. We sequentially carried out two delivery experiments with the nutrient flow set at 100 and $50 \mu \mathrm{L} \cdot \mathrm{min}^{-1}$, while keeping the drug flow rate constant at $1 \mu \mathrm{L} \cdot \mathrm{min}^{-1}$. Fluorescence microscopy revealed that the nuclei became fluorescent when exposed to DAPI, due to the formation of the fluorescent DNA-DAPI complex (see Figure 4). The fluorescence demonstrated that the injected chemical does indeed reach the bottom of the flow cell.

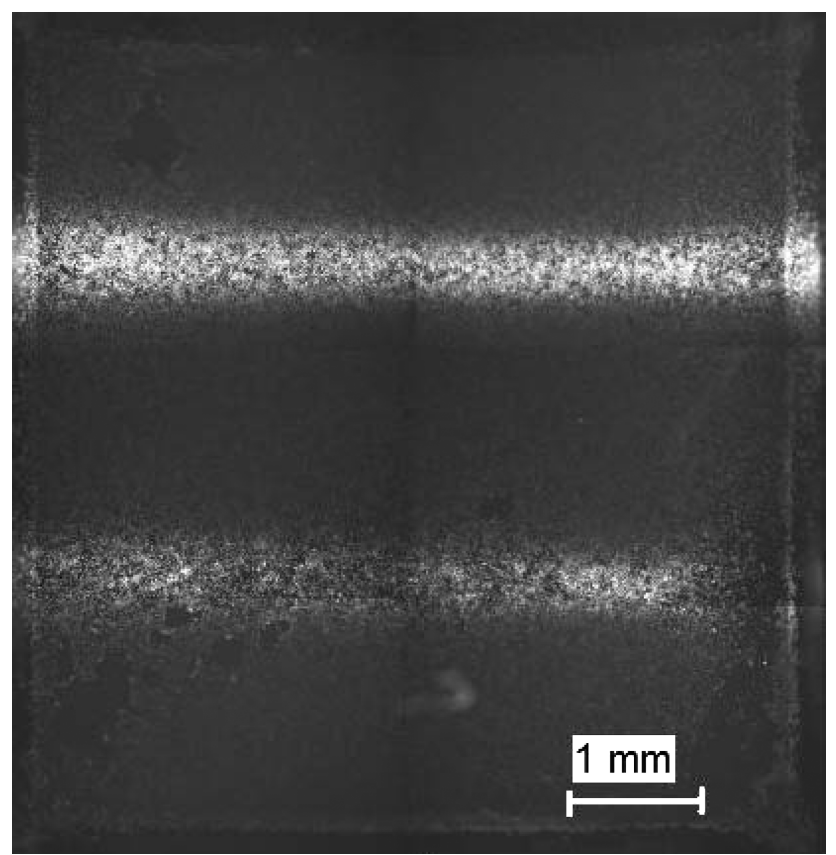

Fig. 4 Experimental validation of drug delivery using cardiomyocytes stained with the DNA stain DAPI. The cells were grown in the $0.6 \times$ $0.6 \mathrm{~cm}^{2}$ cell cultivation chamber, and the nutrient flow was from left to right. The drug was introduced at a flow rate of $1 \mu \mathrm{L} \cdot \mathrm{min}^{-1}$. Nutrient flow was set at $50 \mu \mathrm{L} \cdot \mathrm{min}^{-1}$ and at $100 \mu \mathrm{L} \cdot \mathrm{min}^{-1}$ for drug delivery experiment done with the upper hole and lower hole, respectively. Fluorescence intensities for both experiments cannot be directly compared owing to photobleaching as a consequence of intermediary observation with a fluorescence microscope 

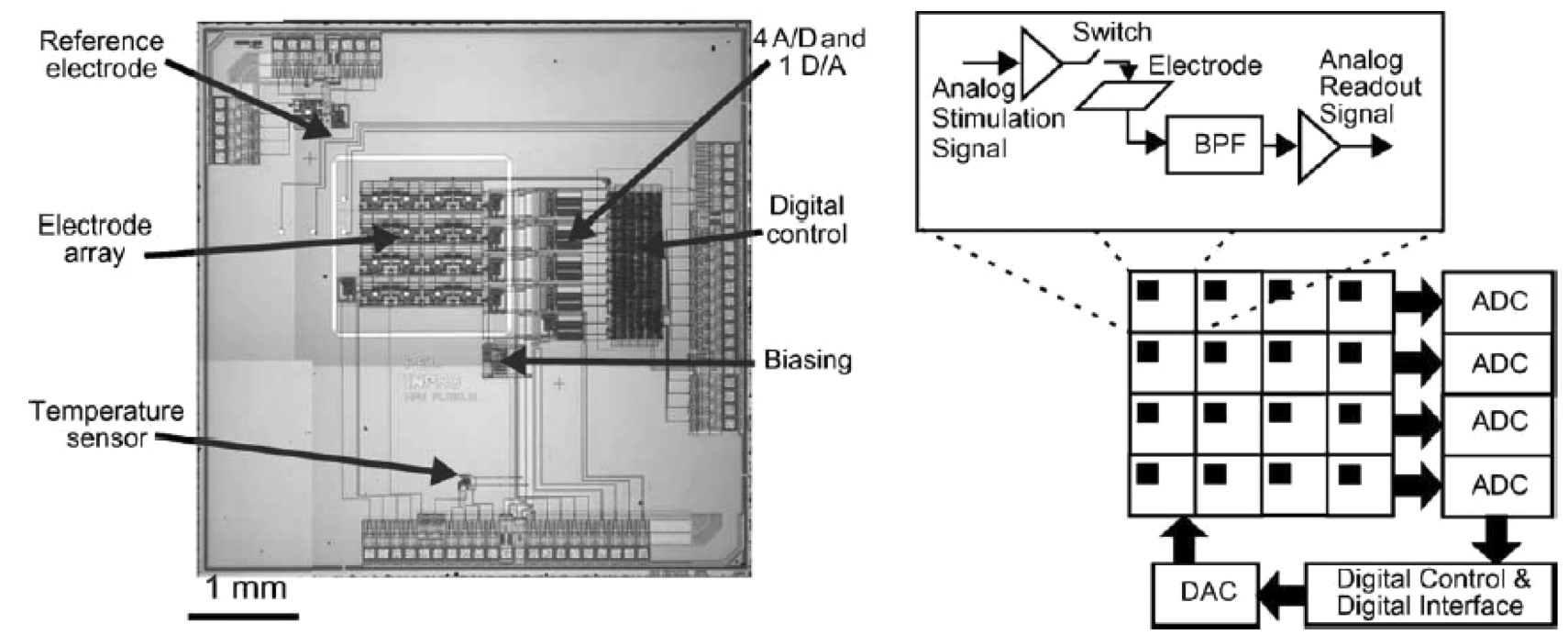

Fig. 5 CMOS chip layout: (left) Picture of the $4.4 \times 4.4 \mathrm{~mm}^{2}$ CMOS chip, and (right) schematic representation of the system architecture

In our first CMOS design, (Heer, et al., 2004) we used an array of $4 \times 4$ electrodes, where each electrode has an associated unit of circuitry which filters and amplifies the recorded electrical signal. Additionally, each electrode can be used to apply electrical stimuli to the cellular culture to elicit an action potential. On-chip multiplexing capabilities translate into an array size that can be increased with minimal design changes. A CMOS chip with 128 electrodes is currently under development. To reduce the number of connections between off-chip instrumentation and the CMOS chip, analog-to-digital and digital-to-analog converters, which enable signal multiplexing, are included. The system architecture also incorporate additional functionalities (reference electrode, temperature sensor, biasing) as illustrated in Fig. 5. We demonstrated that the system can record electric spikes $(\sim 1 \mathrm{mV})$ from active cultures (see Fig. 6 ). To merge the capabilities of microfluidics and CMOS, we investigated several techniques for bonding the CMOS chip to the silicon flow cell. Based on our preliminary tests, we selected flipchip bonding, and are currently testing the bonding of the CMOS to the flow cell. The findings that the 'drug' does indeed reach the HL-1 cells is not expected to significantly change when the glass substrate is replaced with the CMOS MEA.

\section{Conclusion}

We investigated the route to combining the capabilities of microfluidics with that of CMOS bioelectronic chips, and to solve the size mismatch between microfluidic devices and CMOS chips. The ability to control simultaneously the chemical and electrical environments of electrogenic cells is important when studying the influence of chemicals on cellular network activity. The high density arrays of recording/stimulating electrodes available on CMOS chip allow the activity of individual cells within a cellular network to be monitored. This capability will provide neurologists and researchers in life science with instruments to better understand the functioning of the brain, and other tissues that have electroactive cells, such as in the heart and the intestine. The approach presented in this contribution integrates important features of microfluidics and microelectronics, which have been individually recognized for their usefulness in cell biology. Compared to other reports (Takayama et al., 2003), the major drawback of our approach is the low resolution for the localized drug delivery, which results from the geometry required for the integration of the CMOS chip inside the microfluidic device. The lateral resolution is, however, suitable for a number of bioassays, though this
Fig. 6 Recording of electrical signals from cells. (a) The transfer function of the bandpass filter, and (b) a typical spike from embryonic chicken cardiac tissue. High frequencies were cut-off at $3 \mathrm{kHz}$
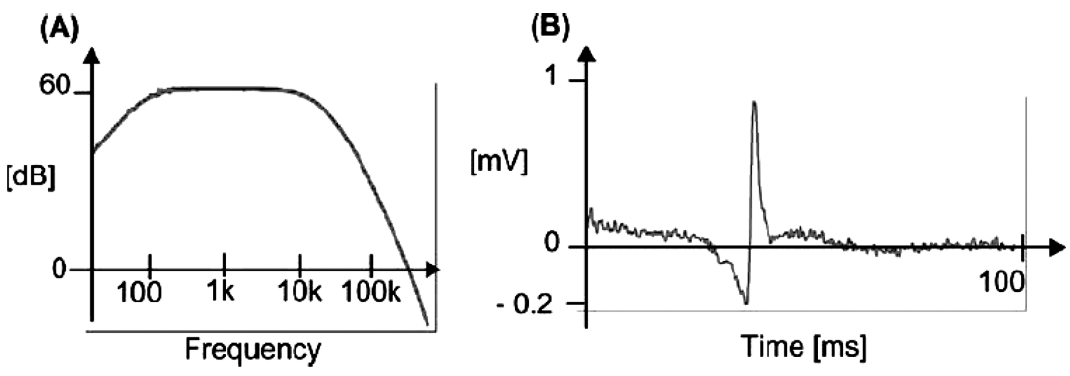
approach will most likely not achieve drug delivery to single cells.

\section{Experimental}

Fabrication of the nutrient delivery system in the flow cell

Flow cells for the cultivation of cells were made in a doubleside polished $100-\mathrm{mm}$ diameter, $525-\mu \mathrm{m}$-thick silicon wafer, using $300 \mathrm{~nm}$ LPCVD silicon nitride as etch mask for two etching steps in $40 \% \mathrm{KOH}$ at $60{ }^{\circ} \mathrm{C}$. The first step consisted of a $425-\mu \mathrm{m}$-deep etch of the silicon to define the area where the cells are to be cultivated. In the second $\mathrm{KOH}$ etching step, $100-\mu \mathrm{m}$-deep entrance and exit channels to the cell cultication area were formed to direct nutrients to the cells. At the same time, the cultivation area was etched further to obtain a through-hole in the silicon. Flow profile measurements described in the first section were performed with flow cells with a $10 \times 10 \mathrm{~mm}^{2}$ cell cultivation chamber. Experiments with cardiac myocytes were performed in $6 \times 6 \mathrm{~mm}^{2}$-sized flow cells. The flow cell was closed at the bottom with a piece of 1.5 -mm-thick Pyrex that has been anodically bonded to the silicon. The top side of the flow cell was closed by a Pyrex cover-plate. As the surface of the cell cultivation area may need to be cleaned on a regular basis, we did not use anodic bonding to close the flow cell from the top side. Instead, silicone grease (polydimethylsiloxane DMS-T63 from ABCR, Karlsruhe, Germany) with a kinematic viscosity of $25 \mathrm{~m}^{2} / \mathrm{s}$ was used to render the structure watertight. Two 1-mm holes for the delivery of nutrients to the cells were drilled ultrasonically in a $100-\mathrm{mm}$ diameter, $500-\mu \mathrm{m}$-thick Pyrex wafer, which was then diced to the size of the flow cell. The flow of nutrients to the cells was controlled by a Harvard PHD2000 syringe pump using a $20 \mathrm{ml}$ plastic syringe.

Fabrication of the drug delivery system in the flow cell

Holes were drilled in the glass coverplate to locally perfuse the cells with drugs, using an Lambda Physics LPX-315 excimer laser at $248 \mathrm{~nm}$ (Lambda Physics, Göttingen, Germany). An energy density of approximately $35000 \mathrm{~mJ} / \mathrm{cm} 2$ at a repetition rate of $100 \mathrm{~Hz}$ was used to drill each opening using 1080 laser pulses ( $\sim 11 \mathrm{~s}$ per hole). The entrance position was continuously purged with helium. The exit hole diameters, used for injection of the drugs, ranged between 40 and $50 \mu \mathrm{m}$, while the inlet openings were about $65 \mu \mathrm{m}$ in diameter. Each hole, 6-8 in total for each flow cell, was connected to a microfluidic channel molded in PDMS (Dow Corning Sylgard 184, Distrelec, Nänikon, Switzerland) using a master produced in SU-8. Tubing (1/16" OD, Ercatech AG, Bern, Switzerland) connecting to the microfluidic channels were inserted into holes punched into the PDMS liquid routing layer using a standard punching tool. The PDMS layer was bonded to the Pyrex cover plate using an oxygen plasma. The flow of the drugs was controlled by a Harvard PHD2000 syringe pump using a $3 \mathrm{ml}$ plastic syringe. To switch between the different drug injection holes, we used an Upchurch sixway valve.

Drug delivery monitored with light

transmission microscopy

Light transmission measurements were performed to investigate the mass transfer properties of the flow cells with liquids dyed with brilliant blue (Fluka, Buchs, Switzerland), a dye that has a diffusion coefficient on the same order of magnitude as the diffusion coefficients of larger pharmaceutical molecules and small proteins. A CCD color camera (JVC TK-C1380) on a Leica MZ 12 microscope was used to obtain images from the reservoir. Videos were recorded during the experiments and digitized with a frame grabber card (VideoGala from PixelSmart, Canada). Light was provided via a glass fibre connected to a Leica CLS 150 light source containing a halogen bulb.

To test the drug injection system, water was pumped continuously at flow rates between 20 and $100 \mu \mathrm{L} / \mathrm{min}$. Two drug injection holes, one located at $0.5 \mathrm{~mm}$ from the flow cell centre ( $4^{\text {th }}$ hole from the top) and one at a distance of $1.87 \mathrm{~mm}$ of its border (hole closest to the edge of the chamber) were used to introduce tracer into the nutrient flow. Brilliant blue solution was injected at $1 \mu \mathrm{L} / \mathrm{min}$. Five minutes were allowed to obtain a stable concentration profile before each measurement.

Drug delivery to the HL-1 cell line

The immortalized HL-1 cardiac myocyte cell line (kindly provided by Dr. W. Claycomb at the Louisiana State University Health Science Center) was used to test the efficacy of the drug delivery system. The cells were maintained in Claycomb Medium (JRH Biosciences, USA) supplemented with $10 \%$ fetal bovine serum (JRH Biosciences, USA), 100 units/mL penicillin (Sigma, USA), $100 \mu \mathrm{g} / \mathrm{mL}$ streptomycin (antibiotic/antimycotic solution, Invitrogen, USA), $0.1 \mathrm{mM}$ norepinephrine (Sigma, USA) and $2.0 \mathrm{mM} \mathrm{L}$ glutamine (Sigma, USA), which was changed approximately every $24 \mathrm{~h}$. Standard incubation conditions of $37^{\circ} \mathrm{C}, 5 \% \mathrm{CO}_{2}$, $95 \%$ air and $95 \%$ relative humidity were used. Prior to plating the cells, the microfluidic device was carefully sterilized with $70 \%$ ethanol. The culture cavity was pretreated with $0.02 \%$ gelatin for $30 \mathrm{~min}$. An initial seed density of $\sim 30,000$ cells per culture cavity was used. Since the culture volume was so small $(\sim 30 \mu \mathrm{L})$ it was necessary to seal the culture while in the incubator to prevent evaporation. A head space over the 
culture was fabricated from a thick frame of PDMS $(15 \times$ $15 \mathrm{~mm}^{2}$ ), which was placed around the glass culture cavity, and a glass cover slip as the lid. The PDMS did not entirely cover the inlet and outlet channels, thereby allowing for gas exchange between the head space over the culture and the controlled incubator environment.

Immediately preceding the drug delivery experiment, the cells were fixed using 4\% paraformaldehyde in phosphate buffered saline (PBS), and the membrane was permeabilized with $0.2 \%$ Triton X-100 (AppliChem, Germany). Membrane permeabilization is necessary to allow the stain to reach the appropriate cellular structures. In this work, the stain 4', 6'-diamidine-2 phylindole dihydrochloride (DAPI, Sigma, USA) was prepared with an end concentration of $1 \mu \mathrm{g} / \mathrm{ml}$ in PBS. The drug delivery experiment was performed using DAPI as the 'drug' at a flow rate of $1 \mu \mathrm{L} / \mathrm{min}$ and PBS as the buffer at both 50 and $100 \mu \mathrm{L} / \mathrm{min}$. Finally, fluorescence microscopy was used to determine which regions, if any, had been contacted by the stain long enough to bind to the nuclear DNA. This experiment was relatively simple to perform since the cells were dead, and it was no longer necessary to work under sterile conditions.

\section{System design of the CMOS chip.}

The $4.4 \times 4.4 \mathrm{~mm}^{2}$ chip presented in Fig. 5 consists of a $4 \times$ 4 electrode array with integrated reference electrode. Fabrication was performed using an industrial double-polysilicon, triple-metal, 0.6 $\mu \mathrm{m}$ CMOS process at X-Fab, Dresden, Germany. The electrodes are $40 \times 40 \mu \mathrm{m}^{2}$ with a $250 \mu \mathrm{m}$ pitch. A unit of repeating circuitry comprising a buffer and switch for stimulation, a bandpass filter and buffer for readout, is provided with each electrode (inset, Fig. 5). Additionally, the system architecture incorporates four analog-to-digital converters (ADC, one per row of electrodes) and one digitalto-analog converter (DAC). On-chip multiplexing and A/Dconversion allow for a reduction in the number of bondpads, which facilitates the integration of a large array. A digital control unit scans the array during readout, provides individual electrode addressability for stimulation, and interfaces with the outside world. Simultaneous recording and stimulation at each electrode can be performed. For the purpose of monitoring the chip operation temperature and the culture temperature, a temperature sensor has been included.

\section{Electrode design}

Due to the limited selection of materials available in a commercial CMOS process, the electrode material as received from the foundry is aluminum, known to be toxic to neuron. A simple 2-mask post-processing procedure is employed to cover the $\mathrm{Al}$ electrodes with biocompatible platinum. It is necessary to seal the underlying $\mathrm{Al}$ to avoid both poisoning and undesirable electrochemistry that occurs when the $\mathrm{Al}$ comes into contact with the neuron medium. The electrode metal consists of $50 \mathrm{~nm} \mathrm{TiW}$, an adhesion promoter, and $270 \mathrm{~nm}$ of Pt. The metals are sputter-deposited and structured in a lift-off process. An alternating $\mathrm{Si}_{3} \mathrm{~N}_{4} / \mathrm{SiO}_{2}$ passivation stack consisting of $1 \mu \mathrm{m}$ of $\mathrm{Si}_{3} \mathrm{~N}_{4}$, and two times $100 \mathrm{~nm}$ $\mathrm{SiO}_{2}$ and $200 \mathrm{~nm} \mathrm{Si}_{3} \mathrm{~N}_{4}$ (total layer thickness of $1.6 \mu \mathrm{m}$ ) is deposited using plasma-enhanced chemical vapor deposition (PECVD). A reactive-ion etch (RIE) is then used to open the nitride stack defining the size and shape of the electrodes. Detailed information regarding the design of the electronic circuitry was published previously (Heer et al., 2004).

Electric spike recording from cells.

First tests were performed with an in situ preparation of cardiomyocytes from fertilized chicken eggs at embryonic day 10. The hearts were extracted from the embryo, briefly rinsed with cold Dulbecco's PBS (Sigma D-8531, $\mathrm{Ca}^{2+}$ - and $\mathrm{Mg}^{2+}$ -free) and directly transferred onto the electrodes of the chip.

DC-offsets at the electrodes were measured to be up to $1 \mathrm{~V}$, which could cause saturation in the first amplification stage. Since the time-constant of the electrochemistry at the electrode-electrolyte interface is on the order of seconds, we used the on-chip stimulation circuitry to set the electrode to a working point.

Acknowledgments The authors gratefully acknowledge funding from the Information Societies Technology (IST) European Union Future and Emerging Technologies Programme, contract number IST-2000-26463, and the Swiss Bundesamt fuer Bildung und Wissenschaft (BBW), contract number 00.0431.2. The clean-room staff at the University of Neuchâtel is acknowledged for their support in fabricating the flow cells. Prof. Axel Blau is acknowledged for generously providing the cardiac tissue and for helpful discussions.

\section{References}

C.A. Jr. Thomas et al., A miniature microelectrode array to monitor the bioelectric activity of cultured cells. Experimental cell research 74(1), 61-6 (1972).

G.W. Gross et al., The use of neuronal networks on multielectrode arrays as biosensors. Biosensors \& Bioelectronics 10(6/7), 55367 (1995).

G.W. Gross, W.Y. Wen, and J.W. Lin, Transparent indium-tin oxide electrode patterns for extracellular, multisite recording in neuronal cultures. Journal of neuroscience methods 15(3), 243-52 (1985).

E.W. Keefer A. Gramowski, and G.W. Gross, NMDA receptordependent periodic oscillations in cultured spinal cord networks. Journal of Neurophysiology 86(6), 3030-3042 (2001).

E.W. Keefer et al., Characterization of acute neurotoxic effects of trimethylolpropane phosphate via neuronal network biosensors. Biosensors \& Bioelectronics 16(7-8), 513-525 (2001).

A. Tscherter et al., Spatiotemporal characterization of rhythmic activity in rat spinal cord slice cultures. European journal of neuroscience 14(2), 179-90 (2001). 
J. Streit et al., The generation of rhythmic activity in dissociated cultures of rat spinal cord. European journal of neuroscience 14(2), 191202 (2001).

Y. Jimbo and H.P.C. Robinson, Propagation of spontaneous synchronized activity in cortical slice cultures recorded by planar electrode arrays. Bioelectrochemistry 51(2), 107-115 (2000).

Q. Bai and K.D. Wise, Single-unit neural recording with active microelectrode arrays. IEEE transactions on bio-medical engineering 48(8), 911-20 (2001).

B.D. DeBusschere and G.T.A. Kovacs, Portable cell-based biosensor system using integrated CMOS cell-cartridges. Biosensors \& Bioelectronics 16(7-8), 543-556 (2001).

B. Eversmann et al., A $128 \times 128$ CMOS bio-sensor array for extracellular recording of neural activity. Journal of Solid-State Circuits 38(12), 2306-2317 (2004).

A. Tourovskaia X. Figueroa-Masot, and A. Folch, Differentiation-ona-chip, A microfluidic platform for long-term cell culture studies. Lab on a Chip 5(1), 14-19 (2005).

P.J. Hung et al., Continuous perfusion microfluidic cell culture array for highthroughput cell-based assays. Biotechnology and Bioengineering 89(1), 1-8 (2004).
H. Kaji, M. Nishizawa and T. Matsue, Localized chemical stimulation to micropatterned cells using multiple laminar fluid flows. Lab on a Chip 3(3), 208-211 (2003).

S. Takayama et al., Selective Chemical Treatment of Cellular Microdomains Using Multiple Laminar Streams. Chemistry \& Biology 10(2), 123-130 (2003).

Y. Matsubara et al., Application of on-chip cell cultures for the detection of allergic response. Biosensors \& Bioelectronics 19(7), 741-747 (2004).

M.A. Unger et al., Monolithic microfabricated valves and pumps by multilayer soft lithography. Science (Washington, D. C.) 288(5463), 113-116 (2000).

J. Liu, C. Hansen and S.R. Quake, Solving the \"world-to-chip \" interface problem with a microfluidic matrix. Analytical Chemistry 75(18), 4718-4723 (2003).

T. Kraus et al., Microfluidic dispensing system for localized stimulation of cellular networks. Lab on a Chip Submitted (2005).

$\mathrm{H}$. Lu et al., Microfluidic Shear Devices for Quantitative Analysis of Cell Adhesion. Analytical Chemistry 76(18), 5257-5264 (2004).

F. Heer et al., CMOS microelectrode array for the monitoring of electrogenic cells. Biosensors \& Bioelectronics 20(2), 358-366 (2004). 